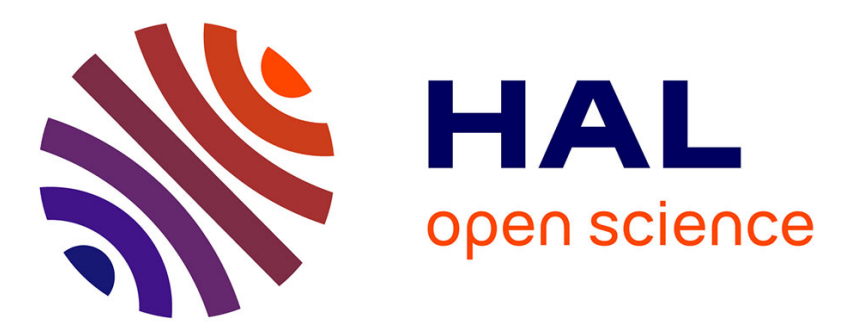

\title{
MoS2-graphene hybrid nanostructures enhanced localized surface plasmon resonance biosensors
}

\author{
Mohamed El Barghouti, Abdellatif Akjouj, Abdellah Mir
}

\section{To cite this version:}

Mohamed El Barghouti, Abdellatif Akjouj, Abdellah Mir. MoS2-graphene hybrid nanostructures enhanced localized surface plasmon resonance biosensors. Optics and Laser Technology, 2020, 130, pp.106306. 10.1016/j.optlastec.2020.106306 . hal-03141656

\section{HAL Id: hal-03141656 \\ https://hal.science/hal-03141656}

Submitted on 18 Aug 2021

HAL is a multi-disciplinary open access archive for the deposit and dissemination of scientific research documents, whether they are published or not. The documents may come from teaching and research institutions in France or abroad, or from public or private research centers.
L'archive ouverte pluridisciplinaire HAL, est destinée au dépôt et à la diffusion de documents scientifiques de niveau recherche, publiés ou non, émanant des établissements d'enseignement et de recherche français ou étrangers, des laboratoires publics ou privés. 


\title{
$\mathrm{MoS}_{2}$-graphene hybrid nanostructures enhanced localized surface plasmon resonance biosensors
}

\author{
Mohamed El barghouti ${ }^{\mathrm{a}, *}$, Abdellatif Akjouj ${ }^{\mathrm{a}, \mathrm{b}}$, Abdellah Mir $^{\mathrm{a}}$ \\ ${ }^{\mathrm{a}}$ Laboratory of Advanced Materials Studies and Applications (LEM2A), Physics Department, Faculty of Science, Moulay Ismail University, B.P. 11201 Zitoune, Meknès, \\ Morocco \\ ${ }^{\mathrm{b}}$ Univ. Lille, Institute of Electronics, Microelectronics and Nanotechnology, UMR CNRS 8520, FST, Department of Physics, 59655 Villeneuve d'Ascq, France
}

We propose a new configuration of a localized surface plasmon resonance (LSPR) biosensor that is based on $\mathrm{MoS}_{2}$-graphene hybrid structures for ultrasensitive detection of molecules. The performance parameters of the proposed biosensor are defined in terms of absorption and sensitivity. Our study show that sensitivity can be greatly increased either by adding a bilayer $\mathrm{MoS}_{2}$ /graphene on the Au nanoparticles or by adding the $\mathrm{MoS}_{2}$ layer or the graphene layer on the surface of the Au nanoparticles. The absorption curves for the proposed LSPR biosensor are analyzed and compared with the conventional biosensors without $\mathrm{MoS}_{2} /$ graphene. By optimizing the structure of the sensor, we find that the sensitivity as high as $360 \mathrm{~nm} / \mathrm{RIU}$ can be achieved with 8-layers of $\mathrm{MoS}_{2}$ and 10-layers of graphene. In addition, we show that the sensitivity can be controlled by changing the number of the monolayer of $\mathrm{MoS}_{2}$ and/or graphene. Finally, we show that this sensor can detect successfully impure water after absorption of target single-stranded DeoxyriboNucleic Acid (ssDNA) biomolecules.

\section{Introduction}

The optical properties of metal nanoparticles and their addressability by spectroscopic techniques have put them at the forefront of the fundamental and applied fields of research, with applications ranging from nanoscale wave guidance to localized surface plasmon resonance (LSPR) biosensor designs, for the biological and chemical detection [1-4]. The understanding of the properties of the gold nanoparticles (AuNPs) is significantly more complex when the AuNPs are deposited on a substrate $[1,5,6]$ and/or when the interaction between the AuNPs and the substrate is not neglected [7-9]. It is quite clear from the theoretical and experimental studies on the AuNPs that several parameters govern their behavior $[10,11]$.

For biosensing applications, the gold-film is not well adapted due to its poor adsorption of biomolecules, which limits the plasmonic biosensor detection properties [12]. A number of studies to amplify the LSPR signal have been demonstrated [10]. For example, LSPR nanostructure with periodic gold nanoparticles (AuNPs) on a substrate has been suggested to improve sensitivity $[12,13]$. Meanwhile, this approach could be used as an effective means of increasing the reaction surface and generating a very confined localized surface plasmon mode, and therefore, enhancing a polarized field-nanoparticle interaction [8]. The AuNP-based LSPR is widely used for the detection of biomolecular elements (Proteins, Antibodies and Nucleic Acids) [13]. However, in order to improve the sensitivity, there are several approaches for improving the adsorption of biomolecules, e.g. the growth of an affinity layer [14,15], using two-dimensional (2D) nano-layers of $\mathrm{MoS}_{2}$ and graphene on AuNPs [16-19].

\footnotetext{
* Corresponding author.

E-mail address: Mohamed.elbarghouti@gmail.com (M. El barghouti).
} 
It is well established that the AuNPs absorb strongly in narrow frequency bands in the visible range that their localized surface plasmon resonances become excited. In addition, the effect of the dielectric environment (surrounding nanoparticles) has been widely studied in the literature [20-23].

Spectacular advances in the control of the optical properties of the leaves of $\mathrm{MoS}_{2}$ materials stimulate the search for new types of twodimensional materials. In fact, monolayers of materials classified as transition-metal dichalcogenides (TDMs) such as $\mathrm{MoS}_{2}$ appeared just recently [24], and are considered to be very promising new type of materials with possible applications in several domains (including bioplasmon detection). Another 2D nanomaterial that entered the picture very recently is the graphene [24] came into the picture because of their extraordinary properties of absorption [25]. Two-dimensional materials are very suitable for adsorption of absorbent materials due to their large surface-to-volume ratio, and their strong conductivity [26]. Elias et al. indicated that the load mobility of the graphene carriers is as high as $106 \mathrm{~cm}^{2} \mathrm{~V}^{-1} \mathrm{~s}^{-1}$ [27]. This is done in the case where the $\mathrm{MoS}_{2}$ layers are deposited on the particles. More specifically, we will examine the effect of progressively varying thickness of the $\mathrm{MoS}_{2}$ layers from $0 \mathrm{~nm}$ to $6.50 \mathrm{~nm}$ (from 0 to 10 layers) and the graphene thickness from $0 \mathrm{~nm}$ to $3.34 \mathrm{~nm}$ ( 0 to 10 layers); the coating of nanoparticles by graphene, it becomes more and more possible, since we know how to bend graphene and $\mathrm{MoS}_{2}$ layers very thin without breaking them; we think that it will be possible to realize our structure.

The semiconductor $\mathrm{MoS}_{2}$, strong light-matter interaction and similarity with the graphene, it is of interest to scientists as a viable alternative to graphene in the manufacture of electronics, especially in photoelectronics [28]. The $\mathrm{MoS}_{2}$ has an absorption of about 5\% per atomic layer [29], whereas the graphene only has an absorption of about $2.3 \%$ per atomic layer $[25,30]$.

Different dielectric layers of the refractive index $\left(\mathrm{n}_{a}\right)$ are deposited on the top of AuNPs/ $\mathrm{MoS}_{2}$ /graphene, which are in turn seated directly on a substrate made of $\mathrm{SiO}_{x}\left(\mathrm{n}_{s}\right)$. Basically, we are numerically studying the nanostructure of how the $\mathrm{MoS}_{2} /$ graphene layers are deposited on AuNPs $\left(\mathrm{SiO}_{x} / \mathrm{AuNPs} / \mathrm{MoS}_{2} /\right.$ graphene). The corresponding geometry is well illustrated in Fig. 1. The incident light (along the y-axis) whose electric field is polarized along the $\mathrm{x}$-axis is also highlighted. In this paper, the geometric parameters of golden nanoparticles will be fixed, as the length of the nanoparticles is $1=125 \mathrm{~nm}, \mathrm{a}=300 \mathrm{~nm}$ is the period parameter (along the $\mathrm{x}$-axis, see Fig. 1 ), and $\mathrm{h}=15 \mathrm{~nm}$ is the height of the nanoparticles.

In our biosensor, we show a significant enhancement of the

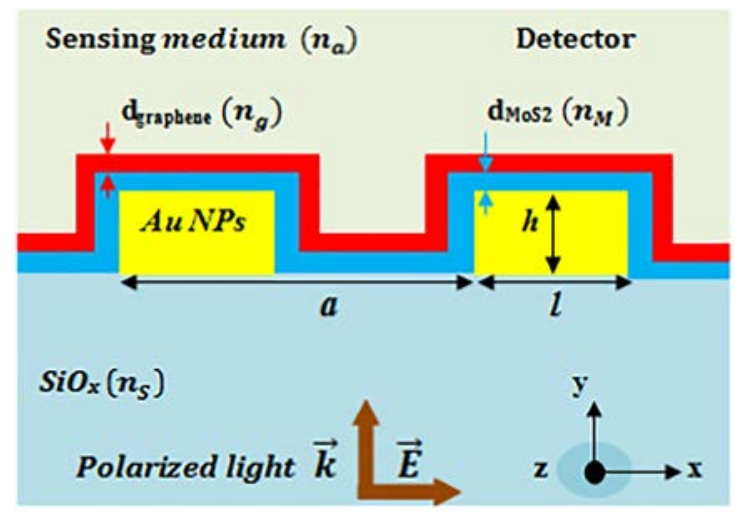

Fig. 1. Schematic representation of AuNPs when covered with few layers of $\mathrm{MoS}_{2}$ /graphene and another dielectric lying on top (detection medium of refraction index $\mathrm{n}_{a}$ ), and deposited on a $\mathrm{SiO}_{x}$ substrate $\left(\mathrm{SiO}_{x} / \mathrm{AuNPs} / \mathrm{MoS}_{2} /\right.$ graphene/dielectric). The particles form a 1D grating along x-axis. The AuNPs grating is characterized by parameter $h$ referring to the particles height, $l$ is their length and $a$ is the lattice parameter. The input wave source (monochromatic plane wave) is also shown. sensitivity of localized surface plasmon resonances of coated AuNPs with layers of $\mathrm{MoS}_{2}$-graphene to the surrounding environment as compared to nanoparticles only deposited on a glass substrate. However, the conventional LSPR biosensor still has a deficiency that its sensitivity not high enough to detect more slight changes in the surrounding environment. Hence, the design of AuNPs/MoS $\mathrm{M}_{2}$-graphene based plasmonic biosensors and like this one defined in Refs. [31-33] with the new types of biosensing $2 \mathrm{D}$ materials. When the refractive index of sensing medium varies from 1.334 to 1.35 , we can obtain the sensitivity of $403.12 \mathrm{~nm} / \mathrm{RIU}$ and $255.64 \mathrm{~nm} / \mathrm{RIU}$ for the conventional LSPR biosensor. Comparing with the nanostructure hybrid biosensor has added a $\mathrm{MoS}_{2}$-graphene layers $(\mathrm{M}=2, \mathrm{~L}=2)$. Indeed, we show that AuNPs coating with layers of $\mathrm{MoS}_{2}$-graphene leads the sensitivity increases to $57.70 \%$. The optimal thickness of $\mathrm{MoS}_{2}$-graphene layers is numerically investigated to achieve a high LSPR sensitive system.

Finally, it should be noted that the excitation system for SPR has to pass through a prism, which makes the structure bulky, whereas the excitation system for LSPR only needs a laser diode to excite the surface plasmons, which makes the device easier to transport and the round also more accessible (the device is cheaper). Another important point for LSPR is that when biological or biochemical fluids interact, effects can be studied at the molecular level. A last point of comparison between SPR and LSPR is that the contact surface with locally detected biological/biochemical molecules is larger with nanoparticles than with metallic film [34].

This paper is organized as follows. Section 2 briefly presents the theoretical model of the simulation and the method followed for calculating the sensitivity of the sensor as well as the absorption coefficient. In Section 3, the design consideration of the proposed LSPR biosensor will be given. Section 4 will be dedicated to the corresponding results for the case where AuNPs are coated with $\mathrm{MoS}_{2}$ /graphene layers. Finally, Section 5 summarizes the main results of this work and compares it with other nanostructures designed for increasing the sensitivity.

\section{Theoretical methods}

\subsection{Simulation model}

The optical properties of gold nanoparticles are determined numerically within a wavelength (in vacuum) domain ranging from visible to near IR, specifically, [400-1200 nm]. Simulations are performed using Finite Element Method (FEM) [35,36]. Actually, the simulation method has already been well documented in Refs.[37-39]; it focus on solving Maxwell's equations in the frequency domain by discretizing space of interest into small finite elements (having various possible shapes such as triangular, mapped, tetrahedral (3D) etc.). Our calculation is carried out in a two-dimensional (2D) domain with Ox and $\mathrm{Oy}$ axes oriented as shown in Fig. 1. An incident plane wave is generated within $\mathrm{SiO}_{x}$ substrate of refraction index $\mathrm{n}_{a}=1.45$, and is launched along the Oy axis (direction of propagation). The plane wave is p-polarized (TM electromagnetic field) TM polarized and its associated electric field vibrates along the Ox axis. The calculation is meant to simulate an infinite array of nanoparticles along Ox axis, and the structure is supposed to be infinite along the direction of Oz. The space discretization is realized by means of free triangular mesh and such that the maximum size of elements is adjusted depending on the material considered. In all dielectrics (layer on top of AuNPs and $\mathrm{SiO}_{x}$ substrate), the elements size is no more than $\left(\frac{\lambda_{0}}{2 n}\right) / 5$, where $\mathrm{n}$ is the index of $\mathrm{SiO}_{x}$ or water/air. In gold particles the skin depth $\delta$ effect has to be taken into account and, as such, at least five points should be set across $\delta$. Within the considered frequency range, $\delta$ lies above $22 \mathrm{~nm}$ (following the calculation method of Ref. [40]), so a maximum elements size of about $3 \mathrm{~nm}$ is sufficient for the numerical calculations to converge. Actually, given that we use (in our structure) thin layers of $\mathrm{MoS}_{2}$ (from $0 \mathrm{~nm}$ to $\approx$ 
Table 1

Gold (Au) Lorentz-Drude model parameters.

\begin{tabular}{ccccc}
\hline Term & $f_{m}[\mathrm{rad} / \mathrm{s}]$ & $\omega_{p}[\mathrm{rad} / \mathrm{s}]$ & $\omega_{m}[\mathrm{rad} / \mathrm{s}]$ & $\Gamma_{m}[\mathrm{rad} / \mathrm{s}]$ \\
\hline $\mathrm{m}=0$ & 0.760 & $13.7210^{15}$ & 0.00 & $0.0805210^{15}$ \\
$\mathrm{~m}=1$ & 0.024 & $13.7210^{15}$ & $0.630510^{15}$ & $0.366110^{15}$ \\
$\mathrm{~m}=2$ & 0.010 & $13.7210^{15}$ & $1.26110^{15}$ & $0.524110^{15}$ \\
$\mathrm{~m}=3$ & 0.071 & $13.7210^{15}$ & $4.51110^{15}$ & $1.32210^{15}$ \\
$\mathrm{~m}=4$ & 0.601 & $13.7210^{15}$ & $6.53810^{15}$ & $3.78910^{15}$ \\
$\mathrm{~m}=5$ & 4.384 & $13.7210^{15}$ & $20.2410^{15}$ & $3.36410^{15}$ \\
\hline
\end{tabular}

$6.5 \mathrm{~nm}$ ) in which light attenuates as well (being a transition metal class material), we decided to fix the maximum elements size at $0.006 \mathrm{~nm}$ (to achieve numerical convergence). This value will also suit the use of a thin layers of graphene (from $0 \mathrm{~nm}$ to $\approx 3.34 \mathrm{~nm}$ ), as a coating for AuNPs/MoS 2 . Obviously, the mesh is relaxed everywhere else as explained above (in dielectric materials).

In Fig. 1, is sketched the simulation models domains in the considered configuration, i.e. when particles are covered with thin layers of $\mathrm{MoS}_{2} /$ graphene. The frequency-dependent complex permittivity of gold metal is described by the Lorentz-Drude model $[41,42]$ :

$\varepsilon(\omega)=\varepsilon_{r, \infty}+\sum_{m=0}^{M} \frac{f_{m} \omega_{p}^{2}}{\omega_{m}^{2}-\omega^{2}+j \omega \Gamma_{m}}$

where $\varepsilon_{r, \infty}$ is the relative permittivity at infinite frequency, $\omega_{p}$ the plasma frequency, and $\omega_{m}, f_{m}$ and $\Gamma_{m}$ are the resonance frequency, ${ }_{2}$ the strength (weight) and the damping rate of the oscillator, respectively. The Lorentz-Drude model uses M damped harmonic oscillators to describe the small resonances observed in the metal's frequency response. The value of the dielectric constant at infinite frequency $\left(\varepsilon_{r, \infty}=1\right)$ and all the other parameters of L-D model are taken from reference [41]. The formers are summarized in Table (1) below:

\subsection{Determination of the sensitivity}

The sensitivity of the refraction index is determined by the following equation [43]:

$S=\frac{\Delta \lambda_{L S P R}}{\Delta n_{a}}\left(n m R I U^{-1}\right)$

where $\mathrm{n}_{a}$ is the refraction index of the detection medium and $\lambda_{L S P R}$ is the resonance wavelength of localized surface plasmon resonance (LSPR) of gold nanoparticles (AuNPs). The detection medium could be either $\mathrm{SiO}_{x}\left(\mathrm{n}_{a}=1.45\right)$ or air $\left(\mathrm{n}_{a}=1\right)$ materials $\left(\Delta n_{a}=0.45\right)$. The sensitivity of the structure arises from the change in refractive index induced by an adsorbate on the interface between the detection medium and the dielectric on top of AuNPs. However, after the point of saturation, a red-shift of plasmonic resonance of $\Delta \lambda_{L S P R}$ resonance has been observed.

\subsection{Calculation of absorption}

In the ATR configuration, according to the principle of energy conservation, the sum of the absorption (A), the Reflection (R) and the transmission ( $\mathrm{T}$ ) must be equal to 1 (i.e., $\mathrm{A}+\mathrm{R}+\mathrm{T}=1$ ). Assuming that there is no energy loss in addition to the absorption of the materials. Under the condition of ATR, R is always close to zero, the absorption of the system can be reduced to $\mathrm{A}=1-\mathrm{R}-\mathrm{T}$. When the LSPR was excited, the absorption gradually increased until it reached a maximum.

The maximum absorption was close to 1 indicating that the incident energy was almost completely absorbed by the stratified materials. Therefore, the maximum incident light energy transfer to the evanescent wave is necessary in order to achieve the best improvement performance of the LSPR [44-47].

\section{Design of the proposed sensors}

We consider a modified biosensor configuration, as shown in Fig. 1. Each layer of the materials is supposed to be piled along the Oy axis. In our nanostructure, $\mathrm{SiO}_{x}$ is chosen as the refraction index coupling substrate $\mathrm{n}_{s}=1.45$, which is deposited on the golden nanoparticles and coated with a MoS 2 layer. The $\mathrm{MoS}_{2}$ layer $\left(\mathrm{d}_{\mathrm{MoS}_{2}}\right)$ is close to the AuNPs, and the other side of the $\mathrm{MoS}_{2}$ layer also connected with a layer of a graphene. The graphene layer $\left(\mathrm{d}_{\text {graphene }}\right)$ is used as a bio-molecular recognition element. We have fixed the geometric parameters of the gold nanoparticles (AuNPs) as the diameter $(1=125 \mathrm{~nm})$, the height $(\mathrm{h}=15 \mathrm{~nm})$ and the interparticle distance $(\mathrm{a}=300 \mathrm{~nm})$. The permittivity of the gold according to the frequency is described by the Lorentz-Drude model Eq. (1).

The complex refractive index $\mathrm{MoS}_{2}$ used is $\mathrm{n}_{M}=5.9+\mathrm{i} 0.8$ $(632 \mathrm{~nm})$ [48], with the thickness of the $\mathrm{MoS}_{2}$ layer being estimated by $\mathrm{d}_{\mathrm{MoS}_{2}}=\mathrm{M} \times 0.65 \mathrm{~nm}$, where $\mathrm{M}$ is the number of $\mathrm{MoS}_{2}$ layers. The graphene is contacted with the detection medium layer in order to increase the capacity of the macromolecular absorption of the biosensor (fixation of the biological element). The thickness of the graphene is varied as $\mathrm{d}_{\text {graphene }}=\mathrm{L} \times 0.34 \mathrm{~nm}$, where $\mathrm{L}$ is the number of graphene layers. The refractive index of the graphene is given by the following formula [49]:

$n_{g}=3.0+i \frac{C_{1}}{3} \lambda$

where the constant $\mathrm{C}_{1}=5.446 \mu \mathrm{m}^{-1}$ [50], and $\lambda$ is the wavelength of the incident light in $\mu \mathrm{m}$. The refractive index of the detection layer is chosen as $\mathrm{n}_{a}=1.000$.

\section{Resultats and discussion}

The operating principle of the proposed biosensor is based on localized surface plasmon resonance (LSPR) produced by interaction of the plasmonic material and the Magnetic Transverse (TM) or P-polarized light incident. A certain wavelength $(\lambda)$ light is passed through the substrate; it is propagated in the substrate-metal interface, which generates an evanescent field. This evanescent field penetrates the gold nanoparticles and continues to propagate with the $\mathrm{K}_{x}$ spread constant along the Ox axis as shown in Fig. 1.

After defining the refractive indices and thicknesses of all the constituent layers of the proposed biosensor, the first aims are to optimize the geometric parameters of the gold nanoparticles and the thicknesses of $\mathrm{MoS}_{2}$ and graphene layers. For the optimization of these different parameters, we calculate the absorption spectrum versus the

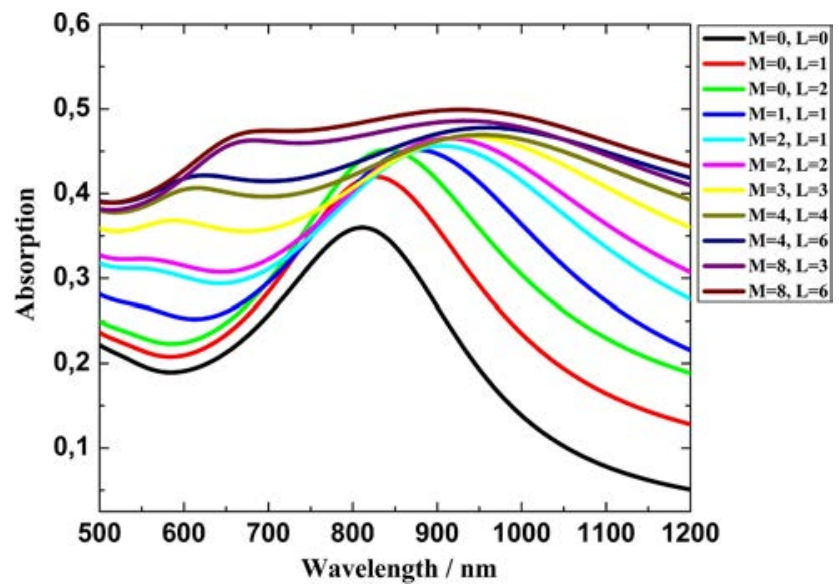

Fig. 2. Absorption curve for conventional LSPR at $(M=0$ and $L=0)$ and for different combinations of $\mathrm{M}$ and $\mathrm{L}$. The nanoparticles are characterized by $1=125 \mathrm{~nm}, \mathrm{~h}=15 \mathrm{~nm}$ and $\mathrm{a}=300 \mathrm{~nm}$. The refractive index of the substrate on which the structure are deposited is $\mathrm{n}_{s}=1.45$. 
wavelength of the incident light. To optimize thicknesses of $\mathrm{MoS}_{2}$ and Graphene, the variations of the LSPR should be as high as possible, while the sensitivity of the biosensor must be as high as possible [22,23].

Fig. 2 presents the absorption spectrum versus wavelength when the incident wave coming from the substrate is normal to the interface. In contrary to the conventional LSPR biosensor [without the $\mathrm{MoS}_{2}$ and graphene layers $(M=0, L=0)]$, the presence of the $\mathrm{MoS}_{2}$ and Graphene in the nanostructure shifts the LSPR and increases the sensibility and the factor of merit. The refractive index $\left(\mathrm{n}_{a}\right)$ value of the sensor detection layer is 1.000 or 1.45 , respectively. At the resonance of LSPR, the absorption intensity must be maximal, which is satisfied by all the curves in this figure. It can be clearly observed from Fig. 2.

We discuss the characteristic sensitivity of the modified biosensor by adding $\mathrm{MoS}_{2}$ and graphene. To illustrate the improvement of the sensitivity performance as shown in Fig. 2 , we set $\mathrm{M}=0$ and $\mathrm{L}=0$, which means the disappearance of the layer of the graphene and the $\mathrm{MoS}_{2}$ layer. It is clearly found that the absorption exists a sharp increase at a certain wavelength range due to the excitation of LSPR. This phenomenon indicates that the incident light is absorbed by the configuration of the biosensor by the exciting LSPR. While the refractive index of the detection layer has small changes due to the molecular interaction $\Delta n_{a}=0.45$, resonance displacement has a small excursion around $\Delta \lambda_{L S P R}=106.22 \mathrm{~nm}$. Therefore, we can obtain the sensitivity $\mathrm{S}=236.5 \mathrm{~nm} / \mathrm{RIU}$ of the structure according to the corresponding calculation of Eq. (2). We can also notice that, for $L \leqslant 3$ and/or $M \leqslant 3$, the variations of the sensitivity remains low (see Table 2). This means that the sensitivity in this case is less than the one without the graphene. In addition, a similar situation occurred when considering the $\mathrm{MoS}_{2}$ layer $(\mathrm{M} \leqslant 2)$ and the graphene sheet $(\mathrm{L} \leqslant 2)$, the variation of resonance LSPR in the east $\Delta \lambda_{L S P R}=97.22 \mathrm{~nm}$ to $(\mathrm{M}=1, \mathrm{~L}=1), \Delta \lambda_{L S P R}$ $=99.61 \mathrm{~nm}$ at $(\mathrm{L}=1, \mathrm{M}=2)$ and $\Delta \lambda_{L S P R}=101.45 \mathrm{~nm}$ at $(\mathrm{L}=2$, $M=2$ ). The sensitivity is $S=216 \mathrm{~nm} / \mathrm{RIU}, \mathrm{S}=221.5 \mathrm{~nm} / \mathrm{RIU}$ and $\mathrm{S}=225.5 \mathrm{~nm} / \mathrm{RIU}$, respectively.

In addition, we also calculated the variation in the LSPR resonance wavelength of the structure by simultaneously adding the $\mathrm{MoS}_{2}$ layer and the graphene layer $(M \geqslant 3$ and $L \geqslant 3$ ) to the higher sensitivity of the proposed biosensor. However, we note that the absorption shift is considerably altered (see Table 2). The resonance LSPR offset is about $\Delta \lambda_{L S P R}=107.28 \mathrm{~nm}$, the sensitivity is $\mathrm{S}=238.40 \mathrm{~nm} / \mathrm{RIU}$ for $\mathrm{M}=3$ and $\mathrm{L}=3$ and for $\Delta \lambda_{L S P R}=157.36 \mathrm{~nm}$ the sensitivity is $\mathrm{S}=349.70 \mathrm{~nm} / \mathrm{RIU}$ for $\mathrm{M}=8$ and $\mathrm{L}=6$.

By comparing the relevant data, we can see that the offset of the resonance wavelength of the biosensor in our structure due to the addition of the $\mathrm{MoS}_{2}$ layer and the graphene layer is greater than the conventional structure of LSPR due to the addition of the graphene layer and the traditional structure of LSPR which contains only the substrate and the gold nanoparticles. Therefore, we can conclude that

Table 2

Evolution of the sensitivity $\mathrm{S}$ acquired theoretically for $\mathrm{SiO}_{x} / \mathrm{AuNPs} / \mathrm{MoS}_{2} /$ graphene, for $(\mathrm{M}=0$ and $\mathrm{L}=0)$ and for different layers of $\mathrm{MoS}_{2}$ and Graphene (M, L) [see Fig. 2].

\begin{tabular}{lllll}
\hline $\begin{array}{l}\mathrm{MoS}_{2} \text { Thickness } \\
(\mathrm{nm})\end{array}$ & $\begin{array}{l}\text { Graphene } \\
\text { Thickness (nm) }\end{array}$ & $\begin{array}{l}\lambda_{L S P R}(\mathrm{~nm}) \\
{\left[\text { Air } \mathrm{n}_{a}=1\right]}\end{array}$ & $\begin{array}{l}\lambda_{L S P R}(\mathrm{~nm}) \\
\left.\mathrm{n}_{a}=1.45\right]\end{array}$ & $\begin{array}{l}\mathrm{S}(\mathrm{nm} / \\
\mathrm{RIU})\end{array}$ \\
\hline 0 & 0 & 810.81 & 917.03 & 236 \\
0 & 0.34 & 826.77 & 929.20 & 227.5 \\
0 & 0.68 & 840 & 945.94 & 235.5 \\
0.65 & 0.34 & 875 & 972.22 & 216 \\
1.30 & 0.34 & 905.17 & 1004.78 & 221.5 \\
1.30 & 0.68 & 913.05 & 1014.50 & 225.5 \\
1.95 & 1 & 937.5 & 1044.78 & 238.5 \\
2.60 & 1.34 & 954.54 & 1065.99 & 247.5 \\
2.60 & 2 & 958.90 & 1082.47 & 274.5 \\
5.20 & 1 & 933.33 & 1076.92 & 319 \\
5.20 & 2 & 925.11 & 1082.47 & 349.5 \\
\hline
\end{tabular}

the sensitivity of the biosensor in our structure $\mathrm{SiO}_{x} / \mathrm{AuNPs} / \mathrm{MoS}_{2} /$ Graphene has a great improvement compared to the traditional simple structure $\mathrm{SiO}_{x} /$ AuNPs.

The refractive index of the detection medium has a significant impact on the absorption intensity and resonance length of LSPR. To determine how LSPR changes with the mean refractive index $\left(\mathrm{n}_{a}\right)$ variation, we calculated the LSPR curves for $\mathrm{n}_{a}=1.000,1.334,1.35,1.37$, 1.40 and 1.45 for the fixed value of $\mathrm{M}=2$ and $\mathrm{L}=2$. The results are described in Fig. 3(a). It can be obvious from the figure that increasing the results of the refractive index of the detection medium increases the wavelength of LSPR. When the refractive index changes from 1.000 to $1.334,1.334$ to $1.35,1.35$ to $1.37,1.37$ to 1.40 and 1.40 to 1.45 , the wavelength of LSPR $\left(\lambda_{L S P R}\right)$ moves from 913.04 to $980.40,980.40$ to 986.84, 986.84 to $993.38,993.38$ to 1000 and 1000 to $1014.50 \mathrm{~nm}$, respectively Fig. 3(b).

In this study, all calculations are performed for the $\mathrm{SiO}_{x}$ substrate having a refractive index of 1.45. It has been reported that the decrease in the refractive index of the functioning sensing medium improves the sensitivity of the sensor [51].

According to the analysis done above, it is clear that the sensitivity of the biosensor can be controlled by the refractive index of detection medium. In order to better understand the enhancement of the sensitivity of the biosensor by the layers of graphene and $\mathrm{MoS}_{2}$, we also discussed the dependence of the sensitivity of the biosensor on the refractive index of detection medium, as shown in Fig. 3. Here we select the refractive index of the layer varies from 1.000 to 1.45 . It can be seen that as the refractive index of detection medium increases by $1.000-1.334$ sensitivity is $\mathrm{S}=201.64 \mathrm{~nm} / \mathrm{RIU}$, for $1.334-1.35$ sensitivity increases significantly $S=403.12 \mathrm{~nm} / \mathrm{RIU}$. For the other refractive index $\left(\mathrm{n}_{a}\right)$ changes from 1.35 to $1.37,1.37-1.40$ and $1.40-1.45$ the sensitivity of $326.76 \mathrm{~nm} / \mathrm{RIU}, 220.75 \mathrm{~nm} / \mathrm{RIU}$ and $289.85 \mathrm{~nm} / \mathrm{RIU}$ respectively.

There is a dominant effect of changing the height of Au nanoparticles from $\mathrm{h}=7$ to $\mathrm{h}=25 \mathrm{~nm}$ on the wavelength of resonance LSPR and the corresponding absorption (drawn in Fig. 4(a)) and sensitivity.

Fig. 4(b) shows the variation of $\Delta \lambda_{L S P R}$ of resonance with the increase in the height of the nanoparticles of gold from 7 to $25 \mathrm{~nm}$. As seen from the figure, the proposed sensor shows the greatest change in wavelength of resonance LSPR to the thickness of $7 \mathrm{~nm}$; therefore, the maximum sensitivity can be obtained at this thickness is $296.18 \mathrm{~nm} /$ RIU (Table 3). For example, the sensitivity is comparatively higher in the thickness varying from 7 to $15 \mathrm{~nm}$. However, to maintain the high detection accuracy we considered the gold layer thickness of $h=15 \mathrm{~nm}$ throughout the analysis.

Another point would be the fluctuating nature of $\lambda_{L S P R}$ from LSPR to $15 \mathrm{~nm}$, suggesting the difficulty in finding a stable detection stage. This phenomenon has also been shown in Fig. 4(a) and (b). At the height of the AuNPs of 7, 10, 15, 20 and $25 \mathrm{~nm}$, the calculated sensitivities are in Table 3, respectively. At optimum height of AuNPs, the thickness of the $\mathrm{MoS}_{2}$ and graphene layers are $\mathrm{M}=2$ and $\mathrm{L}=2$.

Combined with the previous analysis, we deduce that the sensitivity of the biosensor can be enhanced by decreasing the height of the nanoparticles. It is found that the sensitivity of the biosensor increases by $189 \mathrm{~nm} / \mathrm{RIU}$ when the height " $\mathrm{h}$ " of AuNPs moves from 25 to $7 \mathrm{~nm}$.

In Fig. 5, the sensitivity is plotted according to the refractive index of the substrate to the optimized thickness of the $\mathrm{MoS}_{2}$ and graphene layers. The evolution of the sensitivity is plotted at the curve below 8 layers of $\mathrm{MoS}_{2}$ and 6 layers of graphene. The detection medium is suitable for medium $\mathrm{n}_{a}=1.000$ and $\mathrm{n}_{a}=1.45$. Substrate is steep in nature due to the non-existence of solid material for the prism. It is clear from Fig. 5 that the sensitivity increases and decreases with the increase in the refractive index (RI) of substrate $\left(\mathrm{n}_{s}\right)$ [52,53]. Thus, in order to meet the demand for high sensitivity the RI of the substrate should be 1.723 of SF10. If we consider the five well-known substrates i.e. $\mathrm{SiO}_{x}$, $\mathrm{SiO}_{2}$, SF10, ITO and Aspherical the RI with a wavelength of $632.8 \mathrm{~nm}$ 

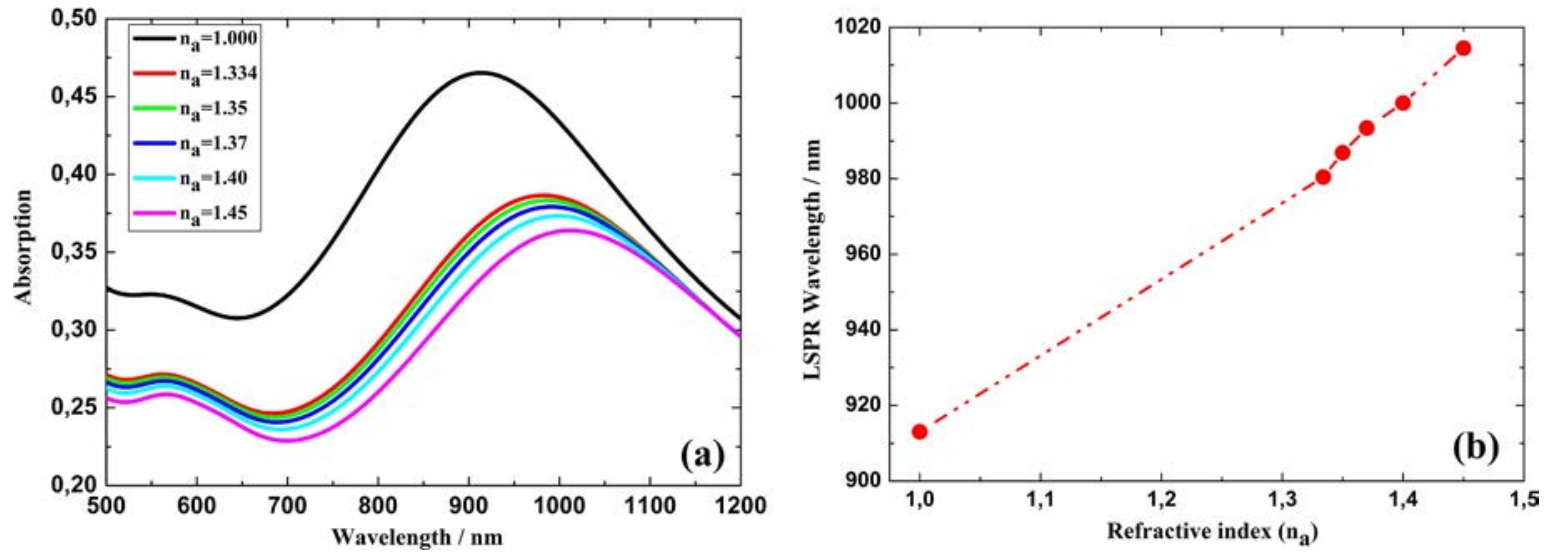

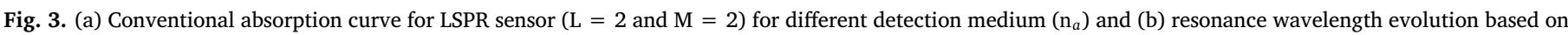
detection medium refractive index.

are $1.45,1.51,1.723,2.00$ and 2.5 respectively, SF10 is the best choice for a better performance of the sensor. For a clearer observation, in Table 4, the LSPR resonance wavelength displacement results for these five substrates are calculated at 1.000 and $1.45 \mathrm{RI}$ of the detection medium and the gold nanostructure parameters are $\mathrm{h}=15 \mathrm{~nm}$, $1=125 \mathrm{~nm}$, and a $=300 \mathrm{~nm}$. Of Table 4 it is clear that the substrate of SF10 has a moderate lag of resonance $\lambda_{L S P R}$ therefore according to Eq. (2) sensitivity will be moderate. Finally, we can notice that if we use SF10 as a substrate, we obtain a high sensitivity, but with a broad resonance peak. If we replace the $\mathrm{SF} 10$ with $\mathrm{SiO}_{x}$, we get a low sensitivity but with a sharp resonance peak, which allows to have a good factor of merit. In the rest of this study, we chose to use $\mathrm{SiO}_{x}$ as a substrate.

In the previous analysis, we verify that the introduction of the $\mathrm{MoS}_{2}$ layer and the graphene layer make a large contribution to the sensitivity of the biosensor LSPR. However, in addition to the introduction of the $\mathrm{MoS}_{2}$ layer (M) and the graphene layer (L), we find that the sensitivity of the biosensor is also sensitive to the number of layers. In order to further improve the sensitivity of the biosensor, we optimize the number of $\mathrm{MoS}_{2}$ layer and the graphene layer. First, we discuss the influence of the number of $\mathrm{MoS}_{2}$ layers (M) on the sensitivity, as shown in Fig. 6(a, b, c and d). It is obvious that the increase in the number of layers of $\mathrm{MoS}_{2}$ layer can improve sensitivity. In addition, the plasmon resonance wavelength moves towards the red with the increase in the number of $\mathrm{MoS}_{2}$ layers, which also implies that the sensitivity of the biosensor can be designed by manipulating the number of layers of $\mathrm{MoS}_{2}$.

Similar tendencies are also well adapted to the influence of the graphene layers, as shown in Fig. 7(a, b, c and d). The sensitivity also
Table 3

Evolution of the theoretically acquired sensitivity for $\mathrm{SiO}_{x} / \mathrm{AuNPs} / \mathrm{MoS}_{2} /$ Graphene at $(M=2, L=2)$ according to the height $(\mathrm{h})$ of the gold nanoparticles, for which $1=125 \mathrm{~nm}$, and a $=300 \mathrm{~nm}$. The refractive index of the substrate on which they are deposited is $\mathrm{n}_{s}=1.45$.

\begin{tabular}{lll}
\hline Gold nanoparticle height $(\mathrm{nm})$ & LSPR wavelength change $(\mathrm{nm})$ & $\mathrm{S}(\mathrm{nm} / \mathrm{RIU})$ \\
\hline 7 & 133.28 & 296 \\
10 & 115.01 & 255.5 \\
15 & 101.45 & 225.5 \\
20 & 84.17 & 187 \\
25 & 48.12 & 107 \\
\hline
\end{tabular}

moves toward larger values with the add number of graphene layer. In addition, the range shift becomes larger with the increase of graphene layers, for example, if one adds 10 layers of graphene $(L=10)$, the sensitivity takes $356 \mathrm{~nm} /$ RIU with a range of increase of $62.5 \mathrm{~nm} / \mathrm{RIU}$.

For the following parameters of the gold nanoparticles $(\mathrm{h}=15 \mathrm{~nm}$, $1=125 \mathrm{~nm}$ and $\mathrm{a}=300 \mathrm{~nm}$ ) and the graphene layer set at $\mathrm{L}=3$ $(1 \mathrm{~nm})$, the resonance wavelength variation in the two detection mediums $\mathrm{n}_{a}=1.000$ and 1.45 with the variation in the number of $\mathrm{MoS}_{2}$ layers is plotted in Fig. 6(a) and (b), respectively. In Fig. 6(a), one observe that $\lambda_{L S P R}$ increases for the $\mathrm{MoS}_{2}$ layers from 0 to 4 and decreases from 4 to 8 . After layer $8, \lambda_{L S P R}$ begins to increase for the structure of the detection medium $\mathrm{n}_{a}=1.000$, this order is followed to increase from 0 to 5 layers for the structure having a detection medium of $\mathrm{n}_{a}=1.45$. After 5 layers of $\mathrm{MoS}_{2}$, the plasmon resonance length remains constant with a different number of $\mathrm{MoS}_{2}$ layers. Fig. 6(d)
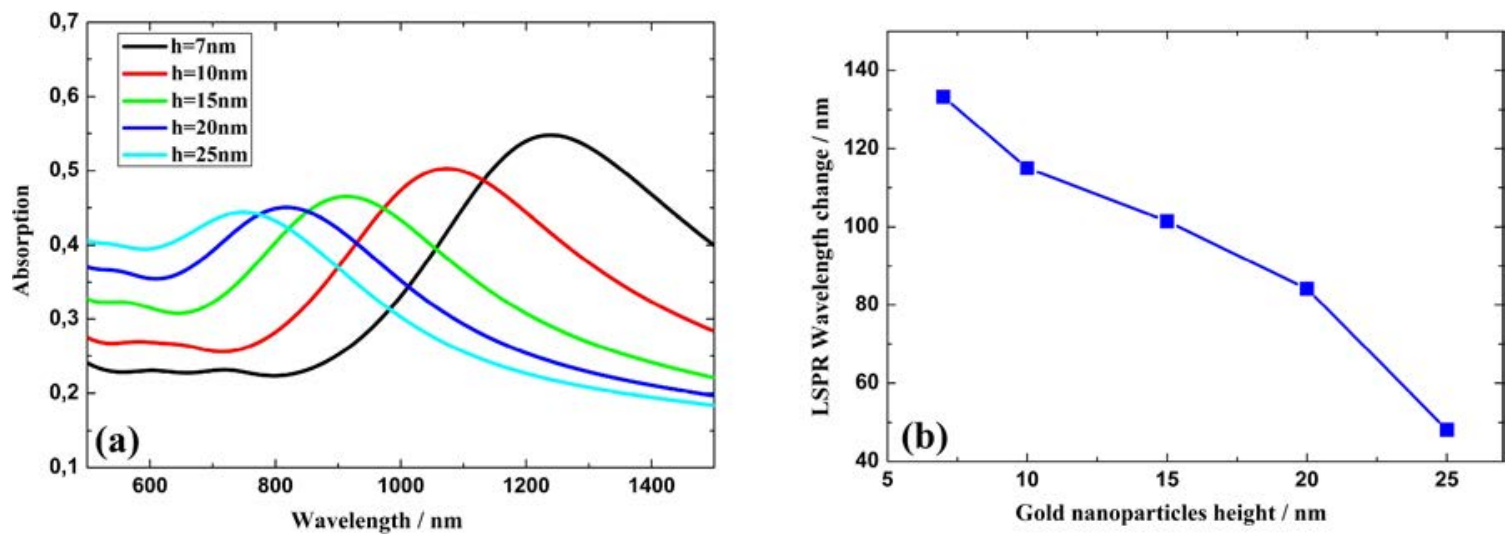

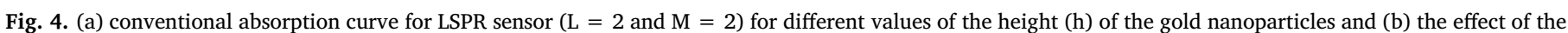

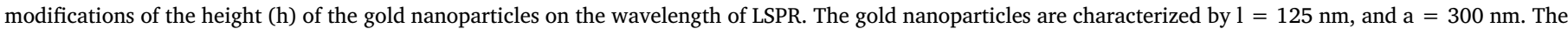
refractive index of the substrate on which they are deposited is $\mathrm{n}_{s}=1.45$. 


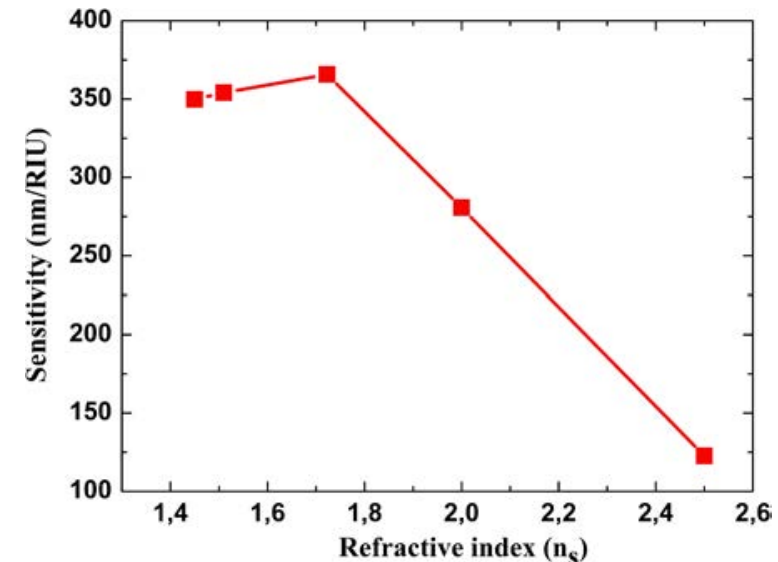

Fig. 5. Evolution of the sensitivity for the structure, Substrate/AuNPs/MoS $/$ Graphene at $(M=8, L=6)$. According to the refractive index of substrate $\left(n_{s}\right)$.

Table 4

Evolution of the sensitivity for the structure, Substrate/AuNPs/ $\mathrm{MoS}_{2} / \mathrm{Graphene}$ to $(M=8, L=6)$ according to the refraction index of the substrate $n_{s}$ (See Fig. 5).

\begin{tabular}{llll}
\hline $\begin{array}{l}\text { Nature of the } \\
\text { substrate }\end{array}$ & $\begin{array}{l}\text { Refractive index } \\
\left(\mathrm{n}_{s}\right)\end{array}$ & $\begin{array}{l}\text { LSPR wavelength } \\
\text { change }(\mathrm{nm})\end{array}$ & $\begin{array}{l}\text { S (nm/ } \\
\text { RIU) }\end{array}$ \\
\hline $\mathrm{SiO}_{x}$ & 1.45 & 157.36 & 350 \\
$\mathrm{Glass}$ & 1.51 & 159.32 & 354 \\
$\mathrm{SF} 10$ & 1.723 & 164.53 & 365.5 \\
ITO & 2.00 & 126.28 & 280.5 \\
Aspherical & 2.5 & 55.14 & 122.5 \\
\hline
\end{tabular}

shows that the sensitivity remains almost constant for $M \leqslant 2$ and increases dramatically for $M>2$. The sensitivity reaches its maximum value for $M=8$, it is equal to $319 \mathrm{~nm} / \mathrm{RIU}$ (see Table 5).

For the parameters of the gold nanoparticles $(\mathrm{h}=15 \mathrm{~nm}$, $\mathrm{L}=125 \mathrm{~nm}$ and $\mathrm{a}=300 \mathrm{~nm}$ ) and of the $\mathrm{MoS}_{2}$ layer fixed to $\mathrm{M}=8$ $(5.20 \mathrm{~nm})$, the resonance wavelength variation in the two detection mediums $\mathrm{n}_{a}=1.000$ and 1.45 as a function of the number of layers of graphene is plotted in Fig. 7(a and b), respectively.

Fig. 7(a) shows that $\lambda_{L S P R}$ decreases for all values of the graphene layers. Fig. $7(\mathrm{~b})$ shows the opposite comportment $\left(\lambda_{L S P R}\right.$ increases with the number of graphene layers), whose resonance wavelength movement begins to increase for the structure shown in Fig. 7(c). One can observe from Fig. 7(d) that the sensitivity increases with the number of graphene layers but for the layers 6,7 and 8 of the graphene the sensitivity remains constant $[14,17,54,55]$ (see Table 6).

We notice in Figs. 7(a) and 7(b) that the resonant wavelength strongly depends on the value that the refractive index ns possesses compared to that of $\mathrm{n}_{a}$. When $\mathrm{n}_{s}>\mathrm{n}_{a}$, we observe a shift of the resonance wavelength towards blue. On the other hand, for $\mathrm{n}_{s}=\mathrm{n}_{a}$, we obtain a spectral shift toward red. These variations are respectively due to the increase in the number of layers of graphene (L) when the number of $\mathrm{MoS}_{2}$ layer set at $\mathrm{M}=8$. In other words, when increasing the number of graphene layers (L), the spectral shift increases.

Comparing the results shown in Figs. 6 and 7; the following conclusions can be drawn: first, the plasmon $\lambda_{L S P R}$ resonance wavelength compensated by the increase in the number of $\mathrm{MoS}_{2}$ layers is greater than the addition of the number of graphene layers [56]. Second, on the basis of the previous discussions, we find that the $\mathrm{MoS}_{2}$ and Graphene layers can be used to improve the sensitivity of the biosensor. In addition, we also illustrate that the optical absorption efficiency of the $\mathrm{MoS}_{2}$ layer is higher than the graphene layer. This property leads to the effect of the $\mathrm{MoS}_{2}$ layer on reflection by increasing the number of layers (M) is greater than that of the graphene (L). Therefore, it has a prior optimization for the number of $\mathrm{MoS}_{2}$ layers of the biosensor. The optimization of the number of graphene layers can be determined after the determination of the number of $\mathrm{MoS}_{2}$ layers. Based on this premise, we first traced the dependence of the sensitivity of the biosensor on the number of $\mathrm{MoS}_{2}$ layers, as shown in Fig. 6(d). The other settings are the same as before. It is clear that the sensitivity of the biosensor is not a monotonous function. We can see that the sensitivity of the biosensor increases first, and then decreases with the increase in the number of $\mathrm{MoS}_{2}$ layers from 1 to 10 , and the maximum sensitivity appears at the 8th layer. The maximum value of the sensitivity of the biosensor is
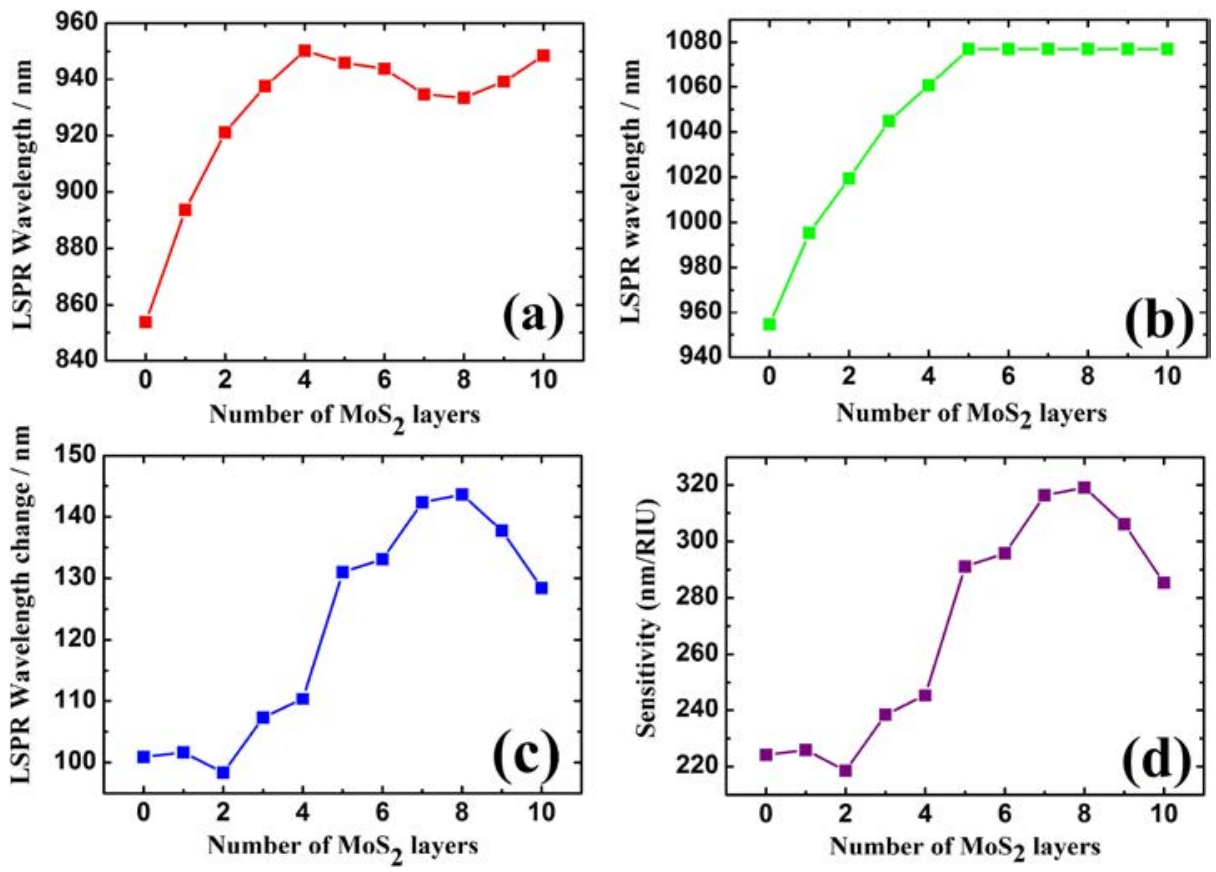

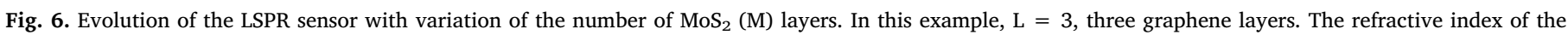

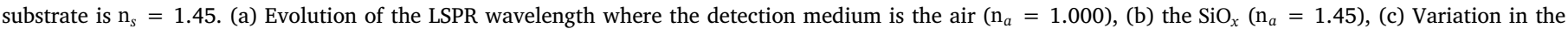

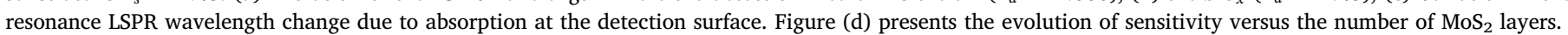



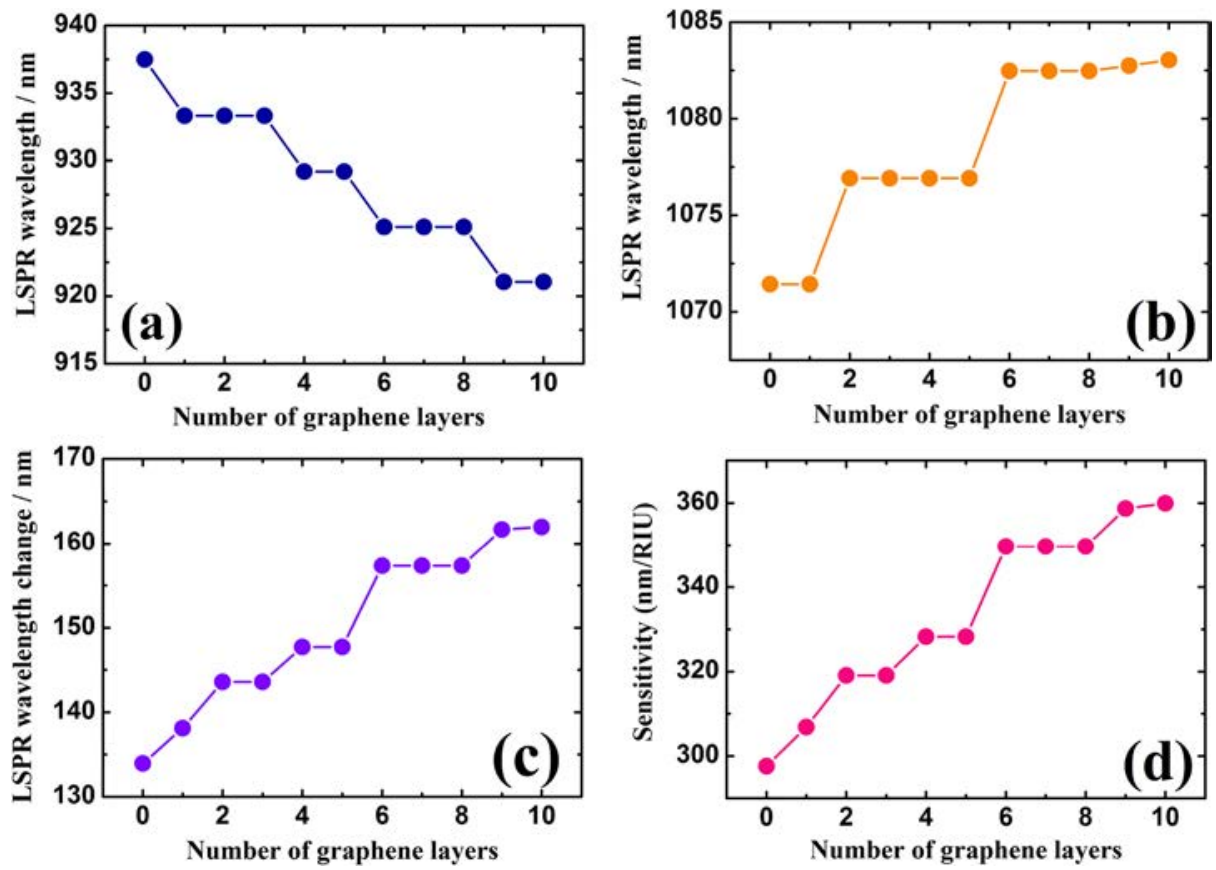

Fig. 7. Plasmon response curves for LSPR sensor versus variation in the number of graphene layers $(\mathrm{L})$ under the condition of three layers of $\mathrm{MoS}_{2}(\mathrm{M}=8)$, (a) evolution of the LSPR wavelength of the structure for the detection medium $\mathrm{n}_{a}=1.000$ (air), (b) for $\mathrm{n}_{a}=1.45$ ( $\mathrm{SiO}_{x}$ ), (c) change in the wavelength LSPR of resonance due to the absorption at the detection surface, and (d) evolution of sensitivity versus $\mathrm{MoS}_{2}$ layers. The refractive index of the substrate on which they are deposited is $\mathrm{n}_{s}=1.45$.

Table 5

Variation of the sensitivity as a function of the number of $\mathrm{MoS}_{2}$ layers with three graphene layers $(\mathrm{L}=3)$.

\begin{tabular}{lllll}
\hline $\begin{array}{l}\mathrm{MoS}_{2}(\mathrm{M}) \\
\text { layer }\end{array}$ & $\begin{array}{l}\mathrm{MoS}_{2} \text { Thickness } \\
(\mathrm{nm})\end{array}$ & $\begin{array}{l}\lambda_{L S P R}(\mathrm{~nm}) \\
{\left[\text { Air } \mathrm{n}_{a}=1\right]}\end{array}$ & $\begin{array}{l}\lambda_{L S P R}(\mathrm{~nm}) \\
\left.\mathrm{n}_{a}=1.45\right]\end{array}$ & $\begin{array}{l}\mathrm{S}(\mathrm{nm} / \\
\mathrm{RIU})\end{array}$ \\
\hline 0 & 0 & 853.66 & 954.54 & 224 \\
1 & 0.65 & 893.62 & 995.26 & 226 \\
2 & 1.30 & 921.05 & 1019.42 & 218.5 \\
3 & 1.95 & 937.5 & 1044.78 & 238.5 \\
4 & 2.60 & 950.23 & 1060.61 & 245.5 \\
5 & 3.25 & 945.94 & 1076.92 & 291 \\
6 & 3.90 & 943.82 & 1076.92 & 296 \\
7 & 4.55 & 934.58 & 1076.92 & 316.5 \\
8 & 5.20 & 933.33 & 1076.92 & 319 \\
9 & 5.85 & 939.18 & 1076.92 & 306 \\
10 & 6.50 & 948.51 & 1076.92 & 285.5 \\
\hline
\end{tabular}

Table 6

Variation of the sensitivity according to the number of graphene layers in the case where the number of layers of $\mathrm{MoS}_{2}$ is $\mathrm{M}=8$.

\begin{tabular}{lllll}
\hline $\begin{array}{l}\text { Graphene (L) } \\
\text { layer }\end{array}$ & $\begin{array}{l}\text { Graphene } \\
\text { Thickness (nm) }\end{array}$ & $\begin{array}{l}\lambda_{L S P R}(\mathrm{~nm}) \\
{\left[\mathrm{Air}_{a}=1\right]}\end{array}$ & $\begin{array}{l}\lambda_{L S P R}(\mathrm{~nm})\left[\mathrm{SiO}_{x}\right. \\
\left.\mathrm{n}_{a}=1.45\right]\end{array}$ & $\begin{array}{l}\mathrm{S}(\mathrm{nm} / \\
\mathrm{RIU})\end{array}$ \\
\hline 0 & 0 & 937.5 & 1071.43 & 298 \\
1 & 0.34 & 933.33 & 1071.43 & 307 \\
2 & 0.68 & 933.33 & 1076.92 & 319 \\
3 & 1 & 933.33 & 1076.92 & 319 \\
4 & 1.34 & 929.20 & 1076.92 & 328.5 \\
5 & 1.68 & 929.20 & 1076.92 & 328.5 \\
6 & 2 & 925.11 & 1082.47 & 349.5 \\
7 & 2.34 & 925.11 & 1082.47 & 349.5 \\
8 & 2.68 & 925.11 & 1082.47 & 349.5 \\
9 & 3 & 921.05 & 1082.74 & 358.5 \\
10 & 3.34 & 921.05 & 1083.03 & 360 \\
\hline
\end{tabular}

about $319.09 \mathrm{~nm} / \mathrm{RIU}$ when $\mathrm{M}=8$. The main reason for this phenomenon is that the rate of light use decreases with the increase in the number of $\mathrm{MoS}_{2}$ layers. Therefore, the number of $\mathrm{MoS}_{2}$ layers cannot be increased indefinitely and it has an optimal value. When we optimize the number of graphene layers, we define $M=8$. We also traced the dependence of the sensitivity of the biosensor on the number of layers of the graphene, as shown in Fig. 6(d). Different from the situation in Fig. 6(d), the sensitivity of the biosensor is a growing and monotonous trend. This suggests that we just need to choose the big one to get a maximum value from the sensitivity of the biosensor [22,57-59].

Generally speaking, after optimization, the maximum sensitivity of the biosensor is about $360 \mathrm{~nm} / \mathrm{RIU}$ with $\mathrm{M}=8$ and $\mathrm{L}=10$. The influence of the number of $\mathrm{MoS}_{2}$ layers and the number of graphene layers on the sensitivity of the biosensor is given in the support information.

One observes a significant enhancement of the sensitivity of localized surface plasmon resonances of coated AuNPs with layers of $\mathrm{MoS}_{2}$ / graphene to the surrounding environment as compared to bare nanoparticles. Hence, the design of Au nanoparticles/ $\mathrm{MoS}_{2}$-graphene basedplasmonic bio-sensors with more sensitivity could be reached. Indeed, we show that AuNPs coating with few layers of $\mathrm{MoS}_{2}$-graphene leads to approximately a $52.54 \%$ higher sensitivity. The optimal thickness of $\mathrm{MoS}_{2}$-graphene layers is numerically investigated to achieve a high LSPR sensitive system.

\section{Detection approach}

This detection concept begins to observe the characteristic absorption according to the wavelength based on LSPR before and after adding biomolecules (biosensing). The process of detecting the DNA (DeoxyriboNucleic Acid) hybridization of the probe from the biosensor LSPR based hybrid AuNPs/MoS $/$ /graphene and other molecule is illustrated in Fig. 8. In Fig. 8(a), a five-layer schematic model substrate/ AuNPs/ $\mathrm{MoS}_{2} /$ Graphene/detection medium consisting of probe DNA and PBS (Phosphate Buffer Saline Solution).

We first present how refractive index varies with the change in adsorbed molecules single strand DNA (ssDNA) into double strand DNA (DNA) on the sensor surface, then the refractive index of sensing medium $\left(\mathrm{n}_{a}\right)$ is mathematically modified as $[11,16]$ : 

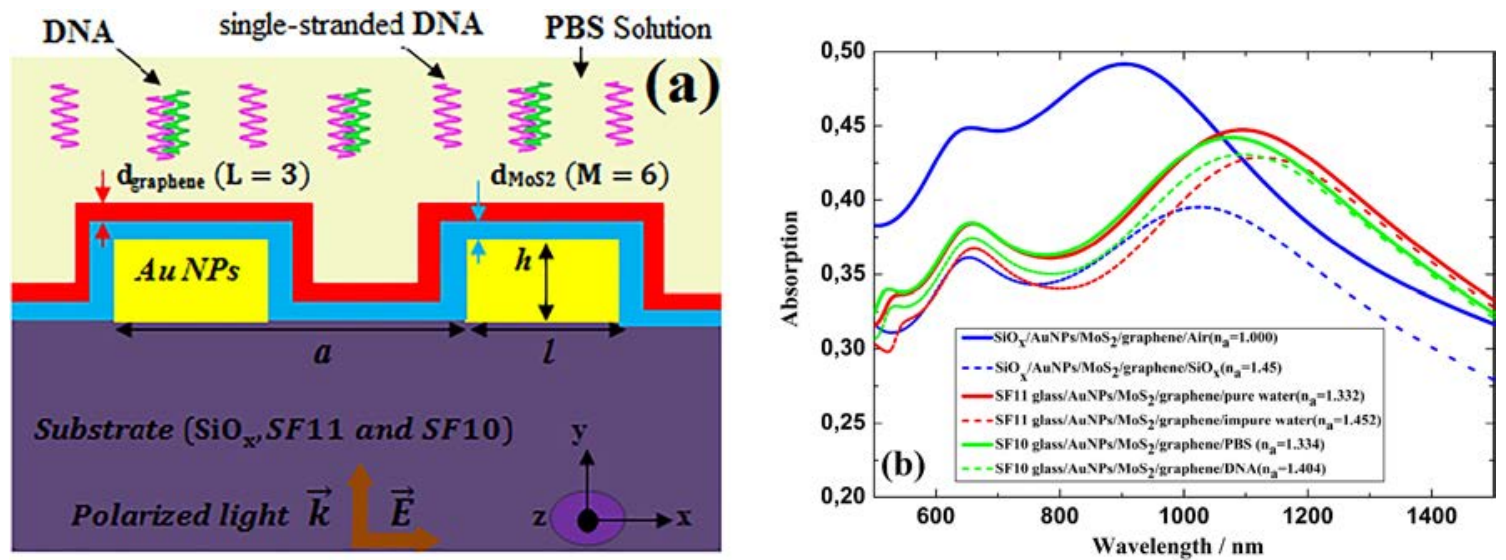

Fig. 8. Detection probe and complementary hybridization of molecule of the $\mathrm{MoS}_{2}$ biosensor project/hybrid LSPR-based graphene. (a) 5-layer model scheme and (b) variation in absorption intensity as a function of wavelength for different structure Substrate/AuNPs/MoS $/{ }_{2} /$ graphene (after addition of different complementary elements to Affinity layer) to ( $\mathrm{M}=6, \mathrm{~L}=3$ ).

$n_{a}(D N A)=n_{a}(P B S)+c_{a} \frac{d n}{d c}$

Here, $\mathrm{n}_{a}$ (DNA) is the RI of the sensing dielectric after adsorption of DNA molecules, $\mathrm{n}_{a}$ (PBS) is RI of the sensing dielectric before adsorption of DNA molecules, $\mathrm{c}_{a}$ is the concentration of adsorbed DNA molecules, and $d n / d c$ is the RI increment due to the adsorbate (DNA). The RI increment parameter is $d n / d c=0.182 \mathrm{~cm}^{3} / \mathrm{gm}$ in the case of using a standard buffer solution [16]. The refractive index of the sensing medium after to add DNA considered in this work is given as:

$n_{a}(D N A)=1.334+\Delta n_{a}$

where $\Delta n_{a}$ is the change of refractive index in sensing medium due to the occurrence of a biological action (DNA). In this study, we assume $\Delta n_{a}=0.07[16,18]$.

The refractive index of detection medium $\left(\mathrm{n}_{a}\right)$ is considered to vary from 1.332 (pure water, before absorption of biomolecules) to 1.452 (impure water, after absorption of the biomolecules) in this study. The proposed LSPR biosensor is shown in Fig. 8(b), which is the comparison between the addition of biomolecules, the red dotted line shows the LSPR curve to add DNA, while the green dotted line represents the LSPR curve to add biomolecules ssDNA (single-stranded DNA) and the black dotted line represents the curve for added a dielectric $\mathrm{SiO}_{x}$. Note that the first mode at about $650 \mathrm{~nm}$ in Fig. 8, is a dipolar mode and is not affected by the nature of the substrate. However, the second mode that appears between $800 \mathrm{~nm}$ and $1000 \mathrm{~nm}$ is clearly a quadrupolar mode (see Figs. 9 and 10). The resonance of this mode is very sensitive to the nature of the substrate. We can notice that the quadrupolar nature of the mode remains insensitive to the nature of the substrate and to the refractive index of the detection medium.

Comparing illustrations in Fig. 8, the following conclusions can be drawn: firstly, the plasmon $\lambda_{L S P R}$ resonance wavelength offset by substrate variation. Secondly, the loss of optical energy caused by the increase in the refractive index of detection for the same substrate.

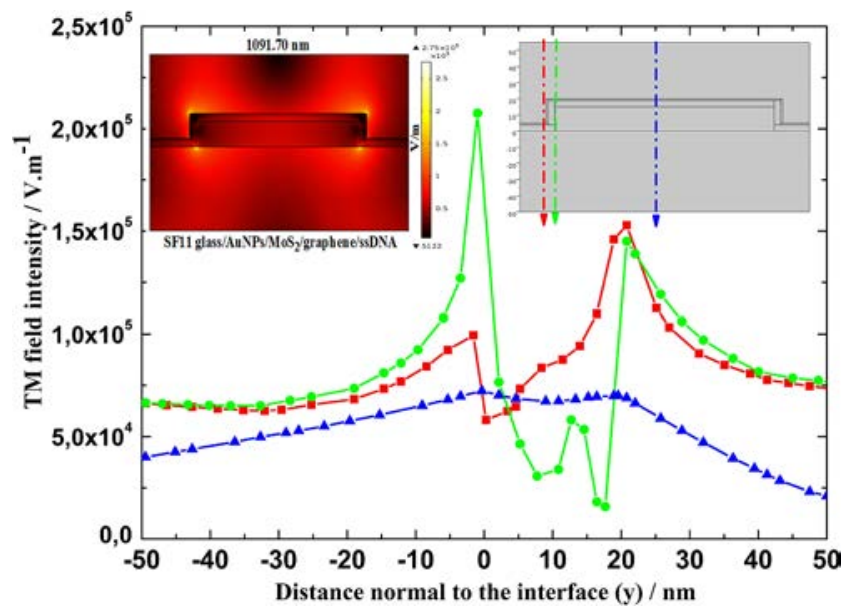

Fig. 10. Intensity of the Transverse magnetic field (TM) according to the normal distance $(y)$ of the interface.

Thirdly, it can be clearly seen from Fig. 8(b) that the sensitivity of the biosensor differs for structural change.

Although $\mathrm{MoS}_{2}$ and Graphene are able to improve the sensitivity and protect the AuNPs of oxidation, it is necessary to see the effect of adding molecules on the performance of the sensor to improve the sensitivity. Therefore, in order to see these affects, comparative the sensors having only " $\mathrm{n}_{a}$ " and " $\mathrm{n}_{s}$ ". For a $\mathrm{SiO}_{x}$ substrate with clearer observations of the $\lambda_{L S P R}$, the curves LSPR to 1.000 and 1.45 are indicated in medallion, the sensitivity is $272 \mathrm{~nm} / \mathrm{RIU}$. For the second structure of a glass SF11 substrate and a detection medium composed of pure water 1.332 and impure water (ssDNA) 1.452, it is clear that the ascending order of sensitivity $300.97 \mathrm{~nm} / \mathrm{RIU}$ can be observed. Same order if for their glass substrate SF10 and the sensitive layer consisting

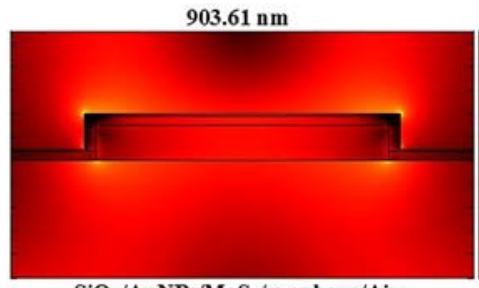

$\mathrm{SiO}_{\mathbf{x}} / \mathrm{AuNPs} / \mathrm{MoS}_{2} / \mathrm{graphene/ \textrm {Ai }}$

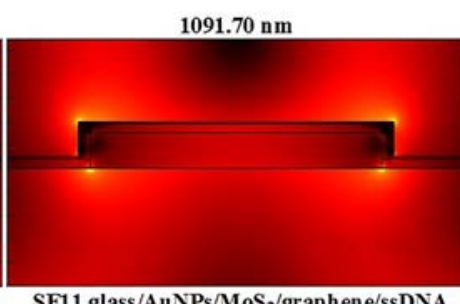

SF11 glass/AuNPs/MoS 2 graphene/ssDNA

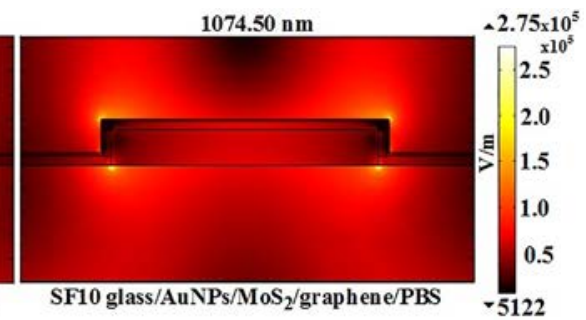

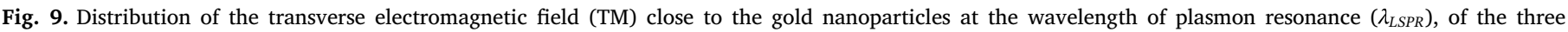
structures of the detection media and of the totally different substrates. 
of PBS, 1.334 and DNA 1.404. This means that the sensitivity $246 \mathrm{~nm} /$ RIU.

This measure, affecting the precision of the biosensor. Thus, we can conclude that one cannot blindly increase the number of $\mathrm{MoS}_{2}$ layers and graphene layers for optimizing the sensitivity of the biosensor but the addition of the biological element improves the sensitivity of sensor LSPR [60].

All the above works are discussed in the case of the geometric parameters of the gold nanoparticles are $\mathrm{h}=15 \mathrm{~nm}, 1=125 \mathrm{~nm}$ and $\mathrm{a}=300 \mathrm{~nm}$ and for six layers of $\mathrm{MoS}_{2}(\mathrm{M}=6)$ and three layers of graphene $(L=3)$. The influence of the different substrate glass and the molecules detected on the sensitivity of the sensor was discussed in the support information.

\section{Intensity of the Transverse magnetic field (TM) for different nanostructures}

The magnetic transverse field (TM) of the p-polarized light is plotted in Fig. 9. An increase in the electromagnetic field strength at the lower corners between the AuNPs and the substrate and at the upper corners between the graphene layer and the detection medium is observed at resonant wavelength when the LSPRs are excited. When the plasmon resonance is excited, the absorption reaches its maximum value and there is an exaltation on the surface of the metal nanoparticles of the electromagnetic field. This exaltation is enhanced at the dielectric/nanoparticle interface by the presence of the $\mathrm{MoS}_{2}$ layer. Moreover, this absorption is reinforced by the $\mathrm{MoS}_{2}$ layer and the graphene layer ( $\mathrm{MoS}_{2} /$ graphene). Therefore, the intensity of the TM field is increased in the detection medium at the corner of the AuNPs corners, which can be seen from Fig. 10.

Thus, in order to detect biomolecules (far from, the intensity AuNPs), the TM field should decrease exponentially, which is also called the evanescent field, in the affinity layer and tends to infinite depth. It can also be said that by increasing the evanescent depth in the sensing medium, the volume of interaction of the evanescent field with the biomolecules is increased which in turn maximizes the sensitivity $[51,52,61]$.

\section{Conclusion}

In conclusion, we have theoretically examined a new type of biosensor structure based on the configuration of a highly sensitive hybrid LSPR sensor AuNPs/MoS $/$ Graphene. The motivation was to study how effectively the structure can detect the hybridization of $\mathrm{MoS}_{2} / \mathrm{Graphene}$ and biomolecules. It is found that the introduction of the $\mathrm{MoS}_{2}$ layer and the graphene layer can significantly improve the sensitivity of the biosensor as the conventional LSPR sensor. Furthermore, we have shown by analysis that the sensitivity of the biosensor can be improved and adjusted by changing the number of $\mathrm{MoS}_{2}$ layer and the graphene layer in the modified structure. The proposed sensor shows an increased sensitivity of the $320 \mathrm{~nm} / \mathrm{RIU}$ and $301 \mathrm{~nm} / \mathrm{RIU}$ in the case of the hybrid layers of $\mathrm{MoS}_{2} /$ Graphene and $\mathrm{MoS}_{2} /$ graphene/ssDNA, respectively. Therefore, it is expected that this proposed biosensor can be used for the detection of biomolecules with a high degree of precision and quality. The proposed biosensor can be fabricated using existing manufacturing technologies. This highly sensitive biosensor can be used for the detection of hybridization, medical diagnosis, enzymatic detection, food security and environmental monitoring.

\section{Author Agreement}

Through all the stages of my study I haven't taken any information or note without acknowledging the source.

\section{Author Statement}

In this work, we propose a new configuration of the localized surface plasmon resonance (LSPR), based on $\mathrm{MoS}_{2} /$ graphene hybrid structures for ultrasensitive bio-detection applications.

Recently, there has been a growing interest in studying light-matter coupling using supported metallic nanostructures arranged into periodic- nanoparticle arrays. The influence of the layer thickness of twodimensional materials such as molybdenum disulfide $\left(\mathrm{MoS}_{2}\right)$ and graphene on the plasmonic behaviour of these arrays has become of great.

In our biosensor, we show a significant enhancement of the sensitivity of localized surface plasmon resonances of coated AuNPs with layers of $\mathrm{MoS}_{2}$ /graphene to the surrounding environment as compared to nanoparticles only deposited on a glass substrate (without $\mathrm{MoS}_{2}$ and graphene). However, the conventional LSPR biosensor still has a deficiency that its sensitivity not high enough to detect more slight changes in the surrounding environment. Hence, the design of AuNPs/ $\mathrm{MoS}_{2}$ graphene based plasmonic biosensors and like this one defined in refs with the new types of biosensing 2D materials.

When the refractive index of sensing medium varies from 1.334 to 1.35 , we obtain the sensitivity of $403.12 \mathrm{~nm} / \mathrm{RIU}$ for our design compared to $255.64 \mathrm{~nm} / \mathrm{RIU}$ obtained for the conventional LSPR biosensor. Comparing with the nanostructure hybrid biosensor has added a $\mathrm{MoS}_{2-}$ graphene layers $(M=2, L=2)$. Indeed, we show that AuNPs coating with layers of $\mathrm{MoS}_{2}$-graphene leads the sensitivity increases to $\mathbf{5 7 . 7 0} \%$. The optimal thickness of $\mathrm{MoS}_{2}$-graphene layers is numerically investigated to achieve a high LSPR sensitive system.

\section{Acnowledgments}

A. Akjouj gratefully acknowledges the hospitality of the department of Physics, Faculty of Science, University of Moulay Ismail of Meknes. This work was partially supported by the program FINCOME "Centre National pour la Recherche Scientifique et Technique, Morocco".

\section{Appendix A. Supplementary material}

Supplementary data associated with this article can be found, in the online version, at https://doi.org/10.1016/j.optlastec.2020.106306.

\section{References}

[1] D.A. Stuart, A.J. Haes, C.R. Yonzon, E.M. Hicks, R.P. Van Duyne, I.E.E. Proc, Detection of biological analytes at physiological conditions using localized surface plasmon resonance spectroscopy, Nanobiotechnol 152 (2005) 13-22.

[2] K. Aslan, J.R. Lakowicz, C.D. Geddes, Plasmon light scattering in biology and medicine: new sensing approaches, visions and perspectives, Curr. Opin. Chem. Biol. 9 (2005) 538-544.

[3] J. Wu, C. Zhou, J. Yu, H. Cao, S. Li, W. Jia, Design of infrared surface plasmon resonance sensors based on graphene ribbon arrays, Opt. Laser Technol. 59 (2014) 99-103.

[4] K.L. Lee, C.W. Lee, W.S. Wang, P.K. Wei, Sensitive biosensor array using surface plasmon resonance on metallic nanoslits, J. Biomed. Opt. 12 (2007) 044023.

[5] M.D. Malinsky, K.L. Kelly, G.C. Schatz, R.P. Van Duyne, Chain length dependence and sensing capabilities of the localized surface plasmon resonance of silver nanoparticles chemically modified with alkanethiol self-assembled monolayers, J. Am. Chem. Soc. 123 (2001) 1471-1482.

[6] J. Zhao, L. Jensen, J. Sung, S. Zou, G.C. Schatz, R.P. Van Duyne, Interaction of plasmon and molecular resonances for rhodamine $6 \mathrm{G}$ adsorbed on silver nanoparticles, J. Am. Chem. Soc. 129 (2007) 7647-7656.

[7] J.N. Anker, W. Paige Hall, O. Lyandres, N.C. Shah, J. Zhao, R.P. Van Duyne, Biosensing with plasmonic nanosensors, Nat. Mater. 7 (2008) 442-453.

[8] E.M. Larsson, J. Alegret, M. Kall, D.S. Sutherland, Sensing characteristics of NIR localized surface plasmon resonances in gold nanorings for application as ultrasensitive biosensors, Nano Lett. 7 (2007) 1256-1263.

[9] G. Kalyushny, A. Vaskevich, M.A. Schneeweiss, I. Rubinstein, Transmission SurfacePlasmon Resonance (T-SPR) measurements for monitoring adsorption on ultrathin gold island films, Chem. Eur. J. 8 (2002) 3849-3857.

[10] E. Galopin, A. Noual, J.N. Jonsson, J.N. Martin, A. Akjouj, Y. Pennec, B.D. Rouhani, R. Boukherroub, S. Szunerits, Short- and long-range sensing using plasmonic nanostructures: experimental and theoretical studies, J. Phys. Chem. C 113 (2009) 15921-15927.

[11] F. Barka-Bouaifel, J. Niedziółka-Jönsson, X. Castel, O. Saison, A. Akjouj, Y. Pennec, 
G. Cooke, Optical and electrochemical properties of tunable host-guest complexes linked to plasmonic interfaces, J. Mater. Chem. 21 (9) (2011) 3006-3013.

[12] L. Wu, H.S. Chu, W.S. Koh, E.P. Li, Highly sensitive graphene biosensors based on surface plasmon resonance, Opt. Express 18 (2010) 14395-1440.

[13] C. Lertvachirapaiboon, A. Baba, S. Ekgasit, C. Thammacharoen, K. Shinbo, K. Kato, F. Kaneko, Gold nanoparticles synthesis used for sensor applications, IEEE Conf. Proc. ISEIM (2014), https://doi.org/10.1109/ISEIM.2011.6826352.

[14] M. El Barghouti, A. Akjouj, A. Mir, Reticular plasmon resonance detection properties of metal nanoparticles, Physica E 110 (2019) 107-114.

[15] J. Homola, Present and future of surface plasmon resonance biosensors, Anal Bioanal Chem. 377 (2003) 528-539.

[16] M.S. Rahman, Md.Sh. Anower, Md.R. Hasan, Md.B. Hossain, Md.I. Haque, Design and numerical analysis of highly sensitive Au- $\mathrm{MoS}_{2}$-graphene based hybrid surface plasmon resonance biosensor, Opt. Commun. 396 (2017) 36-43.

[17] M. El Barghouti, A. Akjouj, A. Mir, Effect of graphene layer on the localized surface plasmon resonance (LSPR) and the sensitivity in periodic nanostructure, Photonic Nanostruct.: Fundam. Appl. 31 (2018) 107-114.

[18] L. Wu, J. Guo, Q. Wang, S. Lu, X. Dai, Y. Xiang, D. Fan, Sensitivity enhancement by using few-layer black phosphorus-graphene/TMDCs heterostructure in surface plasmon resonance biochemical sensor, Sens. Actuators B: Chem. 249 (2017) 542-548.

[19] M. El Barghouti, A. Akjouj, A. Mir, Effect of $\mathrm{MoS}_{2}$ layer on the LSPR in periodic nanostructures, Optik 171 (2018) 237-246.

[20] K.M. Mayer, J.H. Hafner, Localized surface plasmon resonance sensors, Chem. Rev. 111 (2011) 3828-3857.

[21] S. Szunerits, S. Ghodbane, J. Niedziolka-Jonsson, E. Galopin, F. Klauser, A. Akjouj, Y. Pennec, B. Djafari-Rouhani, R. Boukherroub, D. Steinmuller-Nethl, Development and characterization of a diamond-based localized surface plasmon resonance interface, J Phys. Chem. C 114 (2010) 3346-3353.

[22] M. El Barghouti, A. Akjouj, A. Mir, Numerical analysis of $\mathrm{MoS}_{2}$ on the LSPR in periodical nanostructure of $\mathrm{Ag}$ arrays/graphene for sensitive sensors, Int. J. Microwave Opt. Technol. 13 (2018) 454-461.

[23] M. El Barghouti, A. Mir, A. Akjouj, Sabine Szunerits, Modulation of localized surface plasmon resonances of a silver nanoparticle array upon the presence of $\mathrm{MoS}_{2}$ coatings or underlying thin films, Optik 179 (2019) 819-827.

[24] K.S. Novoselov, A.K. Geim, S.V. Morozov, D. Jiang, Y. Zhang, S.V. Dubonos, I.V. Grigorieva, A.A. Firsov, Electric field effect in atomically thin carbon films, Science 306 (2004) 666-669.

[25] R.R. Nair, P. Blake, A.N. Grigorenko, K.S. Novoselov, T.J. Booth, T. Stauber, N.M.R. Peres, A.K. Geim, Fine structure constant defines visual transparency of graphene, Science 320 (2008) 1308.

[26] J.A. Kim, T. Hwang, S.R. Dugasani, R. Amin, R. Kulkarni, S.H. Park, T. Kim, Graphene based fiber optic surface Plasmon resonance for bio-chemical sensor applications, Sens. Actuators B: Chem. 187 (2013) 426-433.

[27] D.C. Elias, R.V. Gorbachev, A.S. Mayorov, S.V. Morozov, A.A. Zhukov, P. Blake, L.A. Ponomarenko, I.V. Grigorieva, K.S. Novoselov, F. Guinea, A.K. Geim, Dirac conesreshaped by interaction effects in suspended graphene, Nat. Phys. 7 (2011) 701-704.

[28] J. Du, Q. Wang, G. Jiang, C. Xu, C. Zhao, Y. Xiang, Y. Chen, S. Wen, H. Zhang, Ytterbium-doped fiber laser passively mode locked by few-layer Molybdenum Disulfide $\left(\mathrm{MoS}_{2}\right)$ saturable absorber functioned with evanescent field interaction, Scient. Rep. 4 (2014) 6346.

[29] J. Guo, S. Li, Z. He, Y. Li, Z. Lei, Y. Liu, W. Huang, T. Gong, Q. Ai, L. Mao, Y. He, Y. Ke, S. Zhou, B. Yu, Near-infrared photodetector based on few-layer $\mathrm{MoS}_{2}$ with sensitivity enhanced by localized surface plasmon resonance, Appl. Surf. Sci. 483 (2019) 1037-1043.

[30] O. Lopez-Sanchez, D. Lembke, M. Kayci, A. Radenovic, A. Kis, Ultrasensitive photodetectors based on monolayer $\mathrm{MoS}_{2}$, Nat. Nano. Techno. 18 (2013) 497-501.

[31] Z. Lin, L. Jiang, L. Wu, J. Guo, X. Dai, Y. Xiang, D. Fan, Tuning and sensitivity enhancement of surface plasmon resonance biosensor with graphene covered Au$\mathrm{MoS}_{2}$-Au films, IEEE Photon. J. 8 (6) (2016) 1-8.

[32] L. Wu, Q. Wang, B. Ruan, J. Zhu, Q. You, X. Dai, Y. Xiang, High performance lossymode resonance sensor based on few-layer black phosphorus, J. Phys. Chem. C 122 (13) (2018) 7368-7373.

[33] T. Xue, W. Liang, Y. Li, Y. Sun, Y. Xiang, Y. Zhang, Z. Dai, Y. Duo, L. Wu, K. Qi, B.N. Shivananju, L. Zhang, X. Cui, H. Zhang, Q. Bao, Ultrasensitive detection of miRNA with an antimonene-based surface plasmon resonance sensor, Nat. Commun. 10 (1) (2019) 1-9.

[34] J. Zhou, T. Yang, J. Chen, C. Wang, H. Zhang, Y. Shao, Two-dimensional nanomaterial-based plasmonic sensing applications: advances and challenges, Coord. Chem. Rev. 410 (2020) 213218.

[35] K. Dossou, M.A. Byrne, L.C. Botten, Finite element computation of grating scattering matrices and application to photonic crystal band calculations, J. Comput. Phys. 219 (2006) 120-143.

[36] J. Li, Q. Ji, S. Chu, Y. Zhang, Y. Li, Q. Gong, K. Liu, Kebin Shi, Tuning the photoresponse in monolayer $\mathrm{MoS}_{2}$ by plasmonic nano-antenna, Scient. Rep. 6 (2016) 23626.

[37] M.W. Knight, N.J. Halas, Nanoshells to nanoeggs to nanocups: optical properties of reduced symmetry core-shell nanoparticles beyond the quasistatic limit, New J. Phys. 10 (2008) 105006.

[38] D.Y. Lei, A.I. Fernández-Domínguez, Y. Sonnefraud, K. Appavoo, R.F. Haglund, J.B. Pendry, S.A. Maier, Revealing plasmonic gap modes in particle-on-film systems using dark-field spectroscopy, ACS Nano 6 (2012) 1380-1386.

[39] Y. Zhan, D.Y. Lei, X. Li, S.A. Maier, Plasmonic Fano resonances in nanohole quadrumers for ultra-sensitive refractive index sensing, Nanoscale 6 (2014) 4705-4715.

[40] R. Qiang, R.L. Chen, J. Chen, Modeling Electrical Properties of Gold Films at Infrared Frequency Using FDTD Method, Int. J Infrared Millimeter Waves 25 (2004) 1263.

[41] A.D. Rakic, A.B. Djuristic, J.M. Elazar, M.L. Majewski, Analysis of optical channel cross talk for free-space optical interconnects in the presence of higher-order transverse modes, Appl. Opt. 37 (1998) 5271-5283.

[42] C.J. Powell, Analysis of optical- and inelastic-electron-scattering data. II. Application to Al, J. Opt. Soc. Am. 60 (1970) 78-93.

[43] E. Galopin, J. Niedziołka-Jonsson, A. Akjouj, Y. Pennec, B. Djafari-Rouhani, A. Noual, R. Boukherroub, S. Szunerits, Sensitivity of plasmonic nanostructures coated with thin oxide films for refractive index sensing: experimental and theoretical investigations, J. Phys. Chem. C 114 (2010) 11769-11775.

[44] S. Zeng, K.-T. Yong, I. Roy, X.-Q. Dinh, X. Yu, F. Luan, A review on functionalized gold nanoparticles for biosensing applications, Plasmonics 6 (2011) 491-506.

[45] R. Verma, B.D. Gupta, R. Jha, Sensitivity enhancement of a surface plasmon resonance based biomolecules sensor using graphene and silicon layers, Sens. Actuators B Chem. 160 (2011) 623-631.

[46] J. Homola, S.S. Yee, G. Gauglitz, Surface plasmon resonance sensors: review, Sens. Actuators B Chem. 54 (1999) 3-15.

[47] A. Lahav, M. Auslender, I. Abdulhalim, Sensitivity enhancement of guided-wave surface-plasmon resonance sensors, Opt. Lett. 33 (2008) 2539-2541.

[48] A. Castellanos-Gomez, N. Agrait, G. Rubio-Bollinger, Optical identification of atomically thin dichalcogenide crystals, Appl. Phys. Lett. 96 (2010) 213116.

[49] M. Bruna, S. Borini, Optical constants of graphene layers in the visible range, Appl. Phys. Lett. 94 (2009) 213116.

[50] R.R. Nair, P. Blake, A.N. Grigorenko, K.S. Novoselov, T.J. Booth, T. Stauber, N.M.R. Peres, A.K. Geim, Fine structure constant defines visual transparency of graphene, Science 320 (2008) 1308.

[51] J.B. Maurya, Y.K. Prajapati, V. Singh, J.P. Saini, R. Tripathi, Performance of graphene- $\mathrm{MoS}_{2}$ based surface plasmon resonance sensor using silicon layer, Opt. Quant. Electron. 47 (2015) 3599-3611.

[52] A. Shalabney, I. Abdulhalim, Electromagnetic fields distribution in multilayer thin film structures and the origin of sensitivity enhancement in surface plasmon resonance sensors, Sens. Actuators A 159 (2010) 24-32.

[53] L. Wu, Y. Jia, L. Jiang, J. Guo, X. Dai, Y. Xiang, D. Fan, Sensitivity improved SPR biosensor based on the $\mathrm{MoS}_{2}$ /graphene-aluminum hybrid structure, J. Lightwave Technol. 35 (2017) 82-87.

[54] A. Verma, A. Prakash, R. Tripathi, Sensitivity enhancement of surface plasmon resonance biosensor using graphene and air gap, Opt. Commun. 357 (2015) $106-112$.

[55] S. Aksimsek, Z. Sun, Graphene-MoS2 Heterostructure Based Surface Plasmon Resonance Biosensor, IEEE, INSPEC (2016) 16336798.

[56] A.K. Mishra, S.K. Mishra, R.K. Verma, Graphene and beyond graphene $\mathrm{MoS}_{2}$ : a new window in surface-plasmon-resonance-based fiber optic sensing, J. Phys. Chem. C 120 (2016) 2893-2900.

[57] J.B. Maurya, Y.K. Prajapati, V. Singh, J.P. Saini, Sensitivity enhancement of surface plasmon resonance sensor based on graphene-- $\mathrm{MoS}_{2}$ hybrid structure with $\mathrm{TiO}_{2}-\mathrm{SiO}_{2}$ composite layer, Appl. Phys. A 121 (2015) 525-533.

[58] L. Wu, Z. Lin, L. Jiang, J. Guo, X. Dai, Y. Xiang, D. Fan, Long-range surface plasmon with graphene for enhancing the sensitivity and detection accuracy of biosensor, IEEE Photon. J. 8 (2016) 1-9.

[59] A. Akjouj, G. Leveque, S. Szunerits, Y. Pennec, B. Djafari-Rouhani, R. Boukherroub, L. Dobrzynski, Nanometal Plasmon Polaritons, Surf. Sci. Rep. 68 (2013) 1-67.

[60] M.B. Hossain, M.M. Rana, DNA hybridization detection based on resonance frequency readout in graphene on Au SPR biosensor, J. Sens. 2016 (2016) 1-8.

[61] L. Wu, J. Guo, X. Dai, Y. Xiang, D. Fan, Sensitivity enhanced by $\mathrm{MoS}_{2}$-graphene hybrid structure in guided-wave surface plasmon resonance biosensor, Plasmonics 13 (1) (2018) 281-285. 